\title{
RELEVÂNCIA DA INVESTIGAÇÃO QUALITATIVA PARA A ELABORAÇÃO DE MATERIAIS EDUCATIVOS EM SAÚDE
}

\author{
Claudenisa Mara de Araújo Vieira1', Sabrina Alaide Amorim Alves², Maria do Socorro Vieira \\ Lopes $^{3}$ e Marlene Menezes de Souza Teixeira ${ }^{4}$ \\ 1,2Universidade Regional do Cariri (URCA), Brasil. mara28araujo@hotmail.com; sabrina1995amorim@gmail.com \\ ${ }^{3}$ Universidade Regional do Cariri (URCA),RENASF/URCA, Brasil. socorrovieira@hotmail.com \\ ${ }^{4}$ Centro Universitário Dr. Leão Sampaio (UNILEÃO), Brasil. marlenesouza@leaosampaio.edu.br
}

\begin{abstract}
Resumo. O estudo tem como objetivo discutir a relevância da investigação qualitativa na clarificação das necessidades dos usuários/cuidadores para elaboração de materiais educativos em saúde. Método: Pesquisa qualitativa a partir de um recorte da dissertação intitulada de cartilha educativa para o manuseio e descarte de resíduos de saúde produzidos no domicilio. Estudo realizado em um município do centro sul do Ceará-Brasil. Participaram do estudo vinte e três usuários/cuidadores. Para coleta de dados foi utilizada uma entrevista semiestruturada. A análise ocorreu mediante a técnica do Discurso do Sujeito Coletivo (DSC). Resultados: O estudo evidencia que os usuários/cuidadores realizam de forma inadequada o manuseio e descarte dos resíduos de saúde. A pesquisa qualitativa permitiu uma maior aproximação com o fenômeno investigado, considerou as dúvidas e necessidades dos usuários/cuidadores, para que a cartilha elaborada possa ser de fácil compreensão, motivadora, adequada culturalmente a realidade dos cuidadores. Conclusão: A elaboração de material educativo balizada pelo método qualitativo permite maior clareza no material elaborado para que o mesmo favoreça a mudança de comportamento e adoção de hábitos de saúde, contribuindo para uma melhor qualidade de vida.
\end{abstract}

Palavras-chave: Pesquisa Qualitativa; Resíduos de Serviços de Saúde; Educação em Saúde.

\section{RELEVANCE OF QUALITATIVE RESEARCH FOR THE PREPARATION OF HEALTH EDUCATIONAL MATERIALS}

\begin{abstract}
The study aims to discuss the relevance of qualitative research in clarifying the needs of users / caregivers for the elaboration of educational health materials. Method: Qualitative research based on an excerpt from the dissertation entitled an educational booklet for the handling and disposal of health waste produced at home. Study carried out in a city in the south of Ceará-Brazil. Twenty-three users / caregivers participated in the study. For data collection, a semi-structured interview was used. Results: The analysis took place using the Collective Subject Discourse (CSD) technique. The study shows that users / caregivers inappropriately carry out the handling and disposal of health waste. The qualitative research allowed a greater approximation with the investigated phenomenon, considered the doubts and needs of users / caregivers, so that the elaborated booklet can be easy to understand, motivating, culturally appropriate to the reality of caregivers. Conclusion: The preparation of educational material based on the qualitative method allows for greater clarity in the material prepared so that it favors the change of behavior and the adoption of health habits, contributing to a better quality of life.
\end{abstract}

Keywords: Qualitative research; Medical Waste; Health education.

\section{INTRODUÇÃO}

Globalmente, a gestão dos Resíduos de Serviços de Saúde (RSS) se apresenta como um desafio ambiental e de saúde pública, principalmente em países populosos e em desenvolvimento como o Brasil. Esta conjuntura deve-se, de forma especial, a elevada 
demanda por procedimentos de saúde, o que acarreta uma maior produção desse tipo de material (Gallotti et al., 2017).

Compreende-se por Resíduos de Serviço de Saúde materiais produzidos em serviços de assistência médica humana ou animal, laboratórios analíticos, centro de zoonoses, necrotérios, funerárias, serviços de medicina em geral, drogarias, distribuidores farmacêuticos, estabelecimentos de ensino e pesquisa na área da saúde, assim como, aqueles provenientes do serviço de atenção domiciliar (Brasil, 2018).

Dessa forma, os desafios para o gerenciamento dos RSS deixaram de centralizar-se apenas no hospital e se estenderam a uma nova modalidade de assistência à saúde, que consiste na atenção domiciliar. A Atenção Domiciliar (AD), refere-se a uma modalidade de atenção à saúde substitutiva e complementar a internação hospitalar que objetiva a redução das extensivas demandas nos serviços de saúde, diminuição da permanência dos indivíduos no hospital e promoção da autonomia dos usuários (Brasil, 2016).

Em meio as dificuldades da atenção domiciliar, destaca-se a carência de conhecimento por parte dos usuários/cuidadores no que se refere ao manuseio e descarte dos RSS produzidos no domicilio, existindo assim, uma lacuna na produção do conhecimento no que se refere à esta temática.

Nesse contexto, ressalta à importância do uso de tecnologias educacionais na saúde como um importante recurso didático pedagógico, possibilitando maior autonomia dos usuários. Nesse prisma, tecnologias educacionais facilitam o processo de aprendizagem por utilizar uma série de recursos, a saber, como imagens, sons, narrativas que contribuiem para uma maior absorção de informações (Silva, Carreiro, \& Mello, 2017).

No que se refere a construção de um material educativo destaca-se à importância e necessidade da realização de uma investigação qualitativa, cuja finalidade é conhecer a percepção, dúvidas e carências do público alvo no que se refere aos fenômenos estudados. Permitindo assim, a produção de conhecimento científico, mediante à abordagem de informações diretas e assertivas que possa compreender a realidade vivenciada pelo objeto no seu contexto social (Silva, Carreiro, \& Mello, 2017).

Sendo assim questiona-se: quais as necessidades, dúvidas e dificuldades que os usuários/cuidadores apresentam no que se refere ao armazenamento, manuseio e descarte 
dos resíduos de saúde produzidos no domicílio? Para responder esta indagação é necessario uma maior aproximação com os usuarios/cuidadores, com isso, objetivou-se discutir a relevância da investigação qualitativa na clarificação das necessidades dos usuários/cuidadores para elaboração de materiais educativos em saúde.

\section{MÉTODO}

Trata-se de um recorte de um trabalho dissertativo intitulado de "cartilha educativa para o manuseio e descarte de resíduos de saúde produzidos no domicilio". O mesmo desenvolveu-se como um estudo metodológico, que de acordo com Polit e Beck (2011), consiste em um tipo de delineamento que possibilita investigar as formas de obtenção dos dados, promovendo uma avaliação sistemática acerca da organização, construção e validação de instrumentos confiáveis.

O percurso metodológico para a construção da cartilha fundamentou-se no modelo metodológico de Echer (2005), que postula quatro etapas para a construção de um material educativo, a saber: 1) Elaboração de um projeto; 2) Sistematização do conteúdo; 3) Construção da cartilha; 4) Validação da cartilha.

A segunda etapa para a construção da cartilha se referiu a sistematização do conteúdo, a mesma contemplou a realização de uma entrevista com o público alvo com a perspectiva de identificar suas dúvidas sobre o manuseio e descarte dos RSS, posteriormente foi realizada uma revisão integrativa de literatura, com o objetivo de identificar nas literaturas renomadas as recomendações para esses processos. Será descrito o desenvolvimento da investigação qualitativa, realizada por meio da entrevista.

Participaram desta etapa usuários/cuidadores que realizavam procedimentos de saúde no domicílio. Adotou-se como critérios de inclusão: ter idade igual ou superior a 18 anos; desenvolver ações de autocuidado que proporcione a geração de RSS; e está admitido no programa de atenção domiciliar ou ser cuidador de usuários que estão admitidos no mesmo. Por outro lado, foram excluídos do estudo, os usuários que apresentaram sanidade mental comprometida.

Participaram do estudo 23 usuários/cuidadores, durante o mês de junho de 2019, por meio da técnica da entrevista. Para a realização da entrevista seguiu-se um roteiro semiestrturado 
direcionado aos usuários/cuidadores em que foi abordado aspectos referentes à percepção que esses têm do manuseio e descarte dos RSS.

A coleta dos dados pautou-se nas seguintes questões norteadoras: Como o senhor(a) realiza, o manuseio e descarte dos RSS? O senhor (a) utiliza alguma forma de proteção ao manusear os RSS? O senhor (a) tem alguma dúvida sobre o manuseio e descarte dos resíduos de saúde?

A aplicação da entrevista foi realizada pela pesquisadora. Inicialmente foi realizado contato prévio para agendamento das entrevistas que foram realizadas no domicilio. Cada entrevista durou em média 20 minutos e todas foram gravadas com o auxílio de um gravador, as respostas foram transcritas sempre ao final do dia de coleta, para que informações importantes para a compreensão dos fenômenos não fossem perdidas.

Para a análise dos dados foi utilizada a técnica do Discurso do Sujeito Coletivo (DSC), a qual segundo Lefevre e Lefevre (2014) fundamenta-se na teoria das representações sociais e busca incorporar a dimensão individual envolvendo-a na dimensão coletiva, sendo utilizado para expressar um pensamento coletivo.

O DSC utiliza três figuras metodológicas para a sua determinação, que são: Expressões Chaves (EC) que consistem em trechos dos discursos que revelam a essência destes, ou seja, aquilo que os participantes buscaram afirmar sobre a temática. As Ideias Centrais (ICs) que são expressões que revelam o sentido das expressões chaves, e as Ancoragens (AC) que são enunciados que contém uma ideologia, uma teoria ou um valor que está explicitamente apresentado (Figueredo, Chiari, \& Goulart, 2013).

A pesquisa foi desenvolvida de acordo com o estabelecido na Resolução 466/12 do Conselho Nacional de Saúde, referente às pesquisas envolvendo seres humanos (Brasil, 2012), e levou em consideração os princípios da ética em pesquisa, que garante a autonomia dos participantes, o direito de liberdade, escolha e privacidade das informações. A referida pesquisa foi submetida à apreciação do Comitê de Ética em Pesquisa (CEP) da Universidade Regional do Cariri, sendo aprovada com o seguinte parecer 3.363.262.

\section{RESULTADOS E DISCUSSÃO}

A Entrevista, possibilitou uma maior aproximação com os usuários/cuidadores, permitindo uma maior clareza do que precisa ser abordado no material educativo para que este 
material produzido a partir de suas dúvidas e fragilidades possam de fato ser de fácil compreensão e colaborem com a aprendizagem destas pessoas para que os cuidados com os residuos produzidos no domicílio sejam adequadamente manuseados. Entretanto, se faz necessario, antes da apresentação dos discursos a caracterização dos participantes em relação ao sexo e idade conforme aponta a Tabela 1.

Tabela 1. Demonstrativo dos participantes da entrevista de acordo com o sexo e idade.

\begin{tabular}{|c|c|c|}
\hline \multicolumn{1}{|c|}{ SEXO } & QUANTIDADE & PORCENTAGEM \\
\hline MASCULINO & 01 & $4 \%$ \\
\hline FEMININO & 22 & $96 \%$ \\
\hline $\begin{array}{c}\text { TOTAL DE } \\
\text { PARTICIPANTES }\end{array}$ & 23 & $100 \%$ \\
\hline FAIXA ETÁRIA & QUANTIDADE & PORCENTAGEM \\
\hline 30 à 40 anos & 05 & $22 \%$ \\
\hline 41 à 50 anos & 07 & $30 \%$ \\
\hline 51 à 65 anos & 11 & $48 \%$ \\
\hline TOTAL DA AMOSTRA & 23 & $100 \%$ \\
\hline
\end{tabular}

Fonte: Pesquisa direta, Crato-Ce, 2020

Nota-se predominância de participantes do sexo feminino correspondendo a $96 \%$ dos entrevistados, de acordo com o estudo de Souza, Pereira e Silva (2018) os cuidados em domicilio são desenvolvidos, majoritariamente, por mulheres no interior de suas famílias, esta realidade se estabelece por que social e culturalmente criou-se a ideia de que a mulher dispõe de habilidades "inatas" para o cuidado como a delicadeza e organização, fazendo com que as mesmas sejam protagonistas nesse processo.

No que se refere a idade dos participantes, a mesma variou de 30 a 65 anos, havendo um predomínio de $48 \%$ da população na faixa etária entre 51 a 65 anos de idade. Esta realidade se desenvolve como reflexo do menor número de filhos das famílias contemporâneas, assim como, do processo de envelhecimento populacional, que faz com que a grande maioria dos cuidadores sejam os próprios cônjuges (Tomomitsu, Perracini, \& Neri, 2014).

Os dados provenientes das entrevistas foram organizados através da técnica do DSC, a entrevista pautou-se na realização de três questões norteadoras, que se seguem: 
* Questão norteadora1: Como o senhor(a) realiza o armazenamento e descarte dos RSS? As respostas suscitaram duas ideias centrais e por conseguinte o desenvolvimento de dois DSC (Quadro 1 e 2).

Quadro 1. Discurso do Sujeito Coletivo 1

\section{IC 1- O descarte dos RSS ocorre através da coleta de lixo urbana}

\section{Discurso do Sujeito Coletivo 1}

Eu separo as coisas dele das nossas, quando eu vou dá banho, trocar a fralda, aplicar a insulina, coloco tudo dentro de um saco separado das nossas coisas, depois, eu deixo no muro até o dia do carro do lixo passar para levar embora. Quando chega o dia, eu coloco tudo lá fora direito, mas é tudo bem amarrado, não faz perigo de se soltar.

Fonte: Pesquisa direta, Crato-Ce, 2020

Diante do discurso apresentado, percebe-se que os usuários/cuidadores não realizam uma importante etapa do processo de manejo dos RSS, que corresponde a segregação, já que, segundo o discurso todos os resíduos gerados são armazenados em um único recipiente. De acordo com a resolução 222/18 da ANVISA a segregação corresponde a primeira e mais importante etapa do manejo dos RSS, e deve ser realizada no momento e local de sua geração, pelo profissional ou pessoa responsável por sua produção.

De acordo com o DSC 1 o armazenamento dos RSS acontece no muro dos domicílios, no entanto, esse tipo de material não deve ser depositado em locais que permitam o alcance de crianças ou animais nem exposição a chuva, o recomendado pela resolução 222/18 é que esse tipo de resíduo seja armazenado em sacos fechados dentro de coletores tampados e em local com pouca movimentação de pessoas e com acesso restrito (Brasil, 2018).

Ainda de acordo com o discurso supracitado, o descarte dos RSS se faz por meio da coleta de lixo urbano, sendo, por conseguinte depositados em lixões a céu aberto. Esta prática vai contra o preconizado na legislação vigente, que postula que a maioria dos RSS devem passar por um tratamento prévio, para posteriormente serem descartados, na perspectiva de reduzir o grau de contaminação desses materiais (Brasil, 2018).

Além do mais, aponta-se que o descarte dos RSS por meio da coleta urbana está ancorada no desenvolvimento de riscos evitáveis de exposição dos trabalhadores da limpeza urbana, 
assim como, do acometimento do meio ambiente pela deposição de material infectado em locais que não estão preparados para recebê-los (Sanches et al., 2018).

Quadro 2. Discurso do Sujeito Coletivo 2

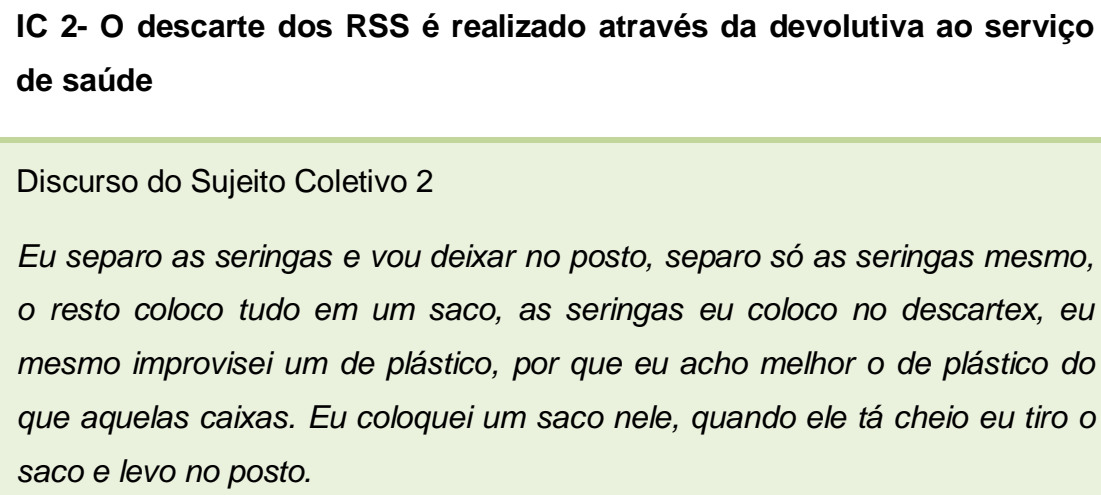

Fonte: Pesquisa direta, Crato-Ce, 2020

Diante do exposto, ressalta-se que o DSC 2 (Quadro 2) está em partes de acordo com o preconizado na legislação vigente, visto que, realiza a separação dos resíduos de acordo com o seu grau de contaminação e capacidade de ocasionar acidentes. Assim como, desencadeia um descarte adequado com a devolução dos materiais perfuro cortantes para o serviço de saúde o que minimiza os agravos ao meio ambiente e reduz a incidência de contaminação (Brasil, 2018).

O Ministério do Trabalho determina que os materiais perfuro cortantes devem ser depositados em recipientes descartáveis ou reutilizáveis que podem ser de papelão, plástico ou metal. Os recipientes reutilizáveis devem ser de plástico ou metal, e devem ser identificados como coletores de perfuro cortantes (Sanches, 2018). De acordo com Matos (2018), as caixas de perfuro cortantes devem ser trocadas sempre que atingirem $2 / 3$ da sua capacidade ou a cada 48 horas.

Entretanto, aponta-se uma fragilidade no comportamento mencionado no discurso, visto que, o discurso afirma retirar os sacos da caixa para realizar o transporte até o serviço de saúde, contudo, esta prática aumenta o risco de acidentes, visto que, facilmente os perfuro cortantes podem perfurar os sacos e contaminarem as pessoas, o recomendado é fazer o descarte de toda a caixa. 
* Questão norteadora 2: O senhor (a) utiliza alguma forma de proteção ao manusear os RSS? As respostas suscitaram duas ideias centrais e por conseguinte o desenvolvimento de dois DSC.

Quadro 3. Discurso do Sujeito Coletivo 3

\section{IC 1- O manuseio dos resíduos de saúde é feito com o uso dos EPI's}

Discurso do Sujeito Coletivo 3

Não é sempre que utilizo alguma coisa, mas as eu uso luva e máscara para me proteger, as vezes uso álcool gel também. O mais importante que eu acho é a luva, sempre que posso não deixo faltar, mas quando não tem uso um saco mesmo, o importante é não se sujar, não é nojo, mas a gente tem que se proteger.

Fonte: Pesquisa direta, Crato-Ce, 2020

O DSC 3 (Quadro 3), evidencia que os usuários/cuidadores desencadeiam um posicionamento correto no que se refere a utilização dos EPI's durante o manuseio dos RSS, vale salientar, que a utilização dos mesmos mostra-se imprescindível para a proteção do usuário, e configura-se como um dos principais pilares de prevenção das doenças infectocontagiosas, visto que, estabelecem uma barreira mecânica que dificultam ou impedem o contato dos microrganismos (Vieira et al., 2015).

Além da utilização dos EPl's destaca a importância da incorporação de outras práticas de biossegurança como a higienização periódica das mãos, visto que, esta conduta protege tanto quem executa o procedimento de saúde quanto quem se beneficia do mesmo, reduzindo o risco de infecção (Vieira et al., 2015).

Quadro 4. Discurso do Sujeito Coletivo 4

\section{IC 2- O manuseio dos resíduos de saúde é feito sem o uso dos EPI's}

Discurso do Sujeito Coletivo 4

Não uso nada para me proteger, não tenho dinheiro para comprar e o serviço de saúde não disponibiliza, eu já tenho muitas despesas, compro fralda, remédio, fruta, infelizmente não sobra dinheiro pra isso.

Fonte: Pesquisa direta, Crato-Ce, 2020 
Diante do exposto, nota-se que parte dos usuários/cuidadores ainda não fazem uso dos EPl's em seu cotidiano (Quadro 4). No universo da atenção domiciliar, o serviço de saúde é o responsável por ofertar os materiais necessários para a continuação da assistência de saúde, assim como, os EPl's para serem utilizados pelos usuários/cuidadores (Alves et al., 2012).

De acordo com a constituição federal de 1988, o estado tem o dever de garantir o atendimento de saúde a toda população brasileira conferindo os insumos básicos para o desenvolvimento das ações de saúde, no entanto, diante da demanda cada vez mais crescente e do sucateamento dos cofres públicos, os recursos financeiros não são suficientes para atender a população da maneira adequada, fazendo com que o usuário tenha que dispor da compra de utensílios, nesse sentido, afirma-se que o fator econômico está diretamente implicado na não utilização dos EPl's pelos usuários/cuidadores (Matos et al., 2018).

* Questão norteadora 3:0 senhor (a) tem alguma dúvida sobre como deve ser realizado o manuseio e descarte dos resíduos de saúde? A mesma suscitou uma ideia central, e um DSC, que correspondeu a:

Quadro 5. Discurso do Sujeito Coletivo 5

\section{IC 1- Dúvidas sobre o manuseio e descarte dos resíduos de saúde}

Discurso do Sujeito Coletivo 5

Nunca tive orientação de como descartar esse tipo de lixo, queria saber se estou fazendo certo em colocar para o carro do lixo levar, e saber a forma correta de fazer esse descarte. Para falar a verdade nunca parei para pensar sobre isso, não tenho dúvida nenhuma, nem sabia que existia um jeito certo, por que na minha cabeça a única coisa que se faz com o lixo é jogar fora, mas eu acho que tá errado né, por que tem que colocar cada coisa no seu canto, mas não me preocupo com essas coisas.

Fonte: Pesquisa direta, Crato-Ce, 2020

O discurso acima citado (Quadro 5) aponta que a principal causa da prática inadequada no que se refere ao manuseio e descarte dos RSS consiste na falta de orientação e capacitação do público alvo. A educação em saúde configura-se como um processo político pedagógico que tem a finalidade de desenvolver um pensamento crítico e reflexivo nas pessoas. Assim como, busca levar a autonomia e emancipação dos indivíduos através do 
aprendizado e da incorporação de ações que os fazem mais conscientes e responsáveis pelos seus atos (Falkenberg et al., 2014).

No referido discurso os participantes comparam os RSS a um simples lixo, demonstrando que não conhecem as particularidades e os riscos provenientes dos mesmos. De acordo com Cafure et al. (2015), os RSS representam um risco em potencial para a população, de maneira direta para aqueles que interagem com esse tipo de resíduo, assim como, de maneira indireta, pelo comprometimento do meio ambiente.

Esse discurso reforça a necessidade de se capacitar e sensibilizar os indivíduos que manuseiam e descarte dos RSS. O uso da entrevista possibilitou identificar as reais necessidades do público-alvo, pôde-se identificar que os mesmos apresentam dificuldades no que se refere a separação, acondicionamento e identificação dos resíduos, e de forma especial, ao descarte dos mesmos. Dessa forma, a referida cartilha foi construída com base nos achados, com o objetivo de ampliar o olhar advindo das falas dos participantes, buscando solucionar dificuldades por meio do que está previsto na literatura vigente.

\section{CONCLUSÃO}

Os materiais educativos se desenvolvem com a perspectiva de suprir necessidade de conhecimento, assim como, favorecer uma mudança de comportamento e adoção de hábitos saudáveis, com isso, a investigação qualitativa através de suas formas de coleta e análise dos dados possibilita a compreensão acerca das principais necessidade do público alvo, fazendo com que o material educativo seja assertivo e aborde aspectos que são relevantes para o processo de educação em saúde, logo, a investigação qualitativa mostrase como um alicerce para a construção dos materiais educativos que sejam facilmente compreendidos, motivadores, que ajudem nos cuidados e que sejam adequados culturalmente.

\section{REFERÊNCIAS}

Alves, S. B., Souza, A. C. S., Rezende, A. F. V. T. K. C., Rezende, F. R., \& Rodrigues, E. G. (2012) Management of waste generated in home care by the Family Health Strategy. Rev Bras Enferm, 65(1), 128-34.

Brasil, Ministério da Saúde (2016). Portaria no 825 de 25 de Abril de 2016. Redefine a Atenção Domiciliar no âmbito do Sistema Único de Saúde (SUS) e atualiza as equipes habilitadas. Brasília: Ministério da Saúde.

Brasil, Ministério da Saúde (2018). Resolução da Diretoria Colegiada-RDC no 222 de 28 de Março de 2018. Regulamenta as Boas Práticas de Gerenciamento dos Resíduos de Serviços de Saúde e dá outras providências. Brasília: Ministério da Saúde. 
Cafure, V. A., \& Graciolli, S. R. P (2015). Residues of health services and their environmental impacts: a bibliographical revision. INTERAÇÕES, 16(2), 301-314.

Escher, I. C (2005). Elaboração de manuais de orientação para o cuidado em saúde. Rev Latino-am Enfermagem, 13(5), 754-757.

Falkernberg, M. B., Mendes, T. P. L., Moraes, E. P., \& Souza, E. M (2014). Health education and education in the health system: concepts and implications for public health. Ciênc. saúde coletiva, 19(3), 243-256.

Figueiredo, M. Z. A., Chiari, B. M., \& Goulart, B. N. G (2013). Discourse of Collective Subject: a brief introduction to a qualitativequantitative research tool*. Distúrb Comun, 25(1), 129-136.

Galloti, F. C. M., Santos, A. T. S., Oliveira, C. B., Passos, \& M. C., Andrade, A. S. A (2017). Gerenciamento de resíduos de serviços de saúde: conhecimento da equipe de enfermagem. Ciências Biológicas e de Saúde Unit., 4(2), 169-184.

Lefevre, F., \& Lefevre, A. M. C (2014). Discurso do sujeito coletivo: representações sociais e intervenções comunicativas. Texto Contexto Enferm, 23(2), 502-507.

Matos, M. C. B., Oliveira, L. B., Queiroz, A. A. F. L. N., Sousa, A. F. L., Valle, A. R. M. C., Andrade, D., \& Moura, M. E. B (2018). Nursing professionals' knowledge regarding the management of waste produced in primary health care. Rev Bras Enferm., 71(6), 113-118.

Polit, D. F., Beck, C. T (2011). Fundamentos de pesquisa em enfermagem:avaliação de evidências para a prática da enfermagem. 7. ed. Porto Alegre: Artmed.

Sanches, A. P. M., Mekaro, K. S., Figueiredo, R. M., \& André, S. C. S (2018). Health-Care Waste: Knowledge of Primary Care nurses. Rev Bras Enferm., 71(5), 2367-2375.

Silva, D. M. L., Carreiro, F. A., \& Mello. R (2017). Educational technologies in nursing assistance in health education: integrating review. Rev enferm UFPE on line., 11(2), 1044-1051.

Souza, I. D., Pereira, J. A., \& Silva, E. M (2018). Entre o Estado, a sociedade e a família: o care das mulheres cuidadoras. Rev Bras Enferm., 71(6), 2882-2890.

Tomomitsu, M. R. S. V., Perracini, M. R., \& Neri, A. L. (2014). Fatores associados à satisfação com a vida em idosos cuidadores e não cuidadores. Ciênc Saúde Coletiva., 19(8), 429-3440.

Vieira, A. N., Lima, D. W. C., Silva, F. T., \& Oliveira, G. W.S (2015). Use of personal protective equipment for nursing professionals in primary health care. Rev enferm UFPE on line, 9(10), 1376-1383. 